PROCEEDINGS OF THE

AMERICAN MATHEMATICAL SOCIETY

Volume 135, Number 2, February 2007, Pages 337-342

S 0002-9939(06)08479-6

Article electronically published on August 4, 2006

\title{
STEINHAUS TILING PROBLEM AND INTEGRAL QUADRATIC FORMS
}

\author{
WAI KIU CHAN AND R. DANIEL MAULDIN
}

(Communicated by Ken Ono)

\begin{abstract}
A lattice $L$ in $\mathbb{R}^{n}$ is said to be equivalent to an integral lattice if there exists a real number $r$ such that the dot product of any pair of vectors in $r L$ is an integer. We show that if $n \geq 3$ and $L$ is equivalent to an integral lattice, then there is no measurable Steinhaus set for $L$, a set which no matter how translated and rotated contains exactly one vector in $L$.
\end{abstract}

\section{INTRODUCTION}

In 1957 Steinhaus proposed the problem that asks whether there exists a set in the plane, which under any rotation and translation contains exactly one point of the integer lattice $\mathbb{Z}^{2}$. This problem was recently solved affirmatively by Jackson and Mauldin in [7] where they detailed a construction of such a set. A natural generalization of Steinhaus' problem is to replace $\mathbb{Z}^{2}$ by a lattice $L$ in the $n$-dimensional Euclidean space $\mathbb{R}^{n}$, where $L=A \mathbb{Z}^{n}$ for some nonsingular $n \times n$ matrix $A$. A set $S$ in $\mathbb{R}^{n}$ is called a Steinhaus set for $L$ if $|(T(S)+x) \cap L|=1$ for all $x \in \mathbb{R}^{n}$ and all isometries $T$. The basic unsolved problem is the following existence problem (see [8, §4]):

Fix $n \geq 2$ and a lattice $L$ in $\mathbb{R}^{n}$. Is there a Steinhaus set for $L$ ?

The existence of Steinhaus sets for all lattices in $\mathbb{R}^{3}$ is still unknown, but the following simple argument [8, page 337] shows that Steinhaus sets for $\mathbb{Z}^{n}, n \geq 4$, do not exist. For, if $S$ were a Steinhaus set for $\mathbb{Z}^{n}(n \geq 4)$, let $x$ and $y$ be the points in $S \cap \mathbb{Z}^{n}$ and $S \cap\left(\mathbb{Z}^{n}+(1 / 2,1 / 2,1 / 2,1 / 2,0 \ldots, 0)\right)$, respectively. It is easy to see that the square-length of $x-y$ is a positive integer. Since every positive integer is a sum of four squares, there will be a vector in $\mathbb{Z}^{n}$ which has the same square-length as $x-y$. This implies that a certain isometric copy of $\mathbb{Z}^{n}$ contains at least two points in $S$, which contradicts the definition of a Steinhaus set.

There are several partial results to the existence problem; see, for instance, 1, [3], 9, [10 and the survey [8. Kolountzakis and Wolff [10] proved that there is no (Lebesgue) measurable Steinhaus set for $\mathbb{Z}^{n}$ for $n \geq 3$. Their approach is simplified in [9, and a slight generalization by Mauldin and Yingst [1] yields some other lattices for which there is no measurable Steinhaus set. Since we shall consider

Received by the editors August 8, 2005 and, in revised form, August 29, 2005.

2000 Mathematics Subject Classification. Primary 11E12, 11H06, 28A20.

Key words and phrases. Representations by quadratic forms, Steinhaus tiling problem.

The research of the first author was partially supported by NSF grant DMS-0138524.

The second author was supported in part by NSF grant DMS-0400481.

(C)2006 American Mathematical Society Reverts to public domain 28 years from publication 
measurable sets, it makes sense to talk about "almost sure" Steinhaus sets, as introduced in [10].

Definition 1.1. A set $S$ is said to have the almost sure Steinhaus property on a lattice $L$ in $\mathbb{R}^{n}$ provided that under almost every isometry $T$ and almost every point $x$ of $\mathbb{R}^{n},|(T(S)+x) \cap L|=1$.

We now describe a sufficient condition, introduced in [11, under which there is no measurable set with the almost sure Steinhaus property on $L$. The volume of the fundamental domain of $\mathbb{R}^{n} / L$ is denoted by $v(L)$. So, if $A$ is an $n \times n$ matrix whose columns form a basis of $L$, then $v(L)$ is equal to $|\operatorname{det} A|$. The dual of $L$, denoted $L^{\#}$, is the set

$$
L^{\#}=\left\{x \in \mathbb{R}^{n}: x \cdot v \in \mathbb{Z} \text { for all } v \in L\right\} .
$$

The set of all possible square-lengths of nonzero vectors in $L$ is denoted as $\mathcal{D}(L)$. Following [1], we say that a lattice $L$ in $\mathbb{R}^{n}$ strongly dominates (resp. weakly dominates) another lattice $M$ in $\mathbb{R}^{n}$, written $L \succ_{s} M\left(\operatorname{resp} . L \succ_{w} M\right)$, if $\mathcal{D}(M) \subseteq$ $\mathcal{D}(L)$ and $v(M) / v(L)$ is irrational (resp. not an integer). It is obvious that $L \succ_{s} M$ implies $L \succ_{w} M$.

Theorem 1.2 ([11, Theorem 1]). Let $L$ be a lattice in $\mathbb{R}^{n}, n \geq 3$, and suppose that there exists a lattice $M$ in $\mathbb{R}^{n}$ where $L^{\#} \succ_{w} M$. Then there is no measurable set with the almost sure Steinhaus property on $L$.

We call two lattices $L$ and $M$ in $\mathbb{R}^{n}$ equivalent if there exists a constant $r$ such that $r L$ is an isometric copy of $M$. It is obvious that if $S$ is a measurable set with the almost sure Steinhaus property on $L$, then $r S$ will be a measurable set with the almost sure Steinhaus property on $r L$. A lattice is called integral if the dot product between any pair of vectors inside the lattice is an integer. The main theorem of this paper is:

Theorem 1.3. Suppose that $L$ is a lattice in $\mathbb{R}^{n}, n \geq 3$, which is equivalent to an integral lattice. Then there is no measurable set with the almost sure Steinhaus property on $L$.

This is a consequence of the following proposition whose proof will be the content of the rest of this paper.

Proposition 1.4. Let $L$ be an integral lattice in $\mathbb{R}^{n}, n \geq 3$. Then there exists a lattice $M$ in $\mathbb{R}^{n}$ such that $L \succ_{s} M$.

Proof of Theorem 1.3. We may assume that $L$ itself is an integral lattice. Then $L^{\#}$ is equivalent to an integral lattice. Choose a real number $r$ such that $r L^{\#}$ is integral. By Proposition 1.4, there exists a lattice $M$ such that $r L^{\#} \succ_{s} M$. By virtue of Theorem 1.2, it suffices to show that $L^{\#} \succ_{s} r^{-1} M$. First, if $a \in \mathcal{D}\left(r^{-1} M\right)$, then $r^{2} a \in \mathcal{D}(M) \subseteq \mathcal{D}\left(r L^{\#}\right)$ and hence $a \in \mathcal{D}\left(L^{\#}\right)$. Next, since $v\left(r L^{\#}\right)=r^{n} v\left(L^{\#}\right)$, then

$$
\frac{v\left(r^{-1} M\right)}{v\left(L^{\#}\right)}=\frac{v(M)}{v\left(r L^{\#}\right)},
$$

which is irrational since $r L^{\#} \succ_{s} M$. 


\section{LATTICES IN QUADRATIC SPACES}

In this section, we briefly review the arithmetic of positive definite integral lattices. Notation and terminology follow those from the book by O'Meara [12.

Let $\mathfrak{o}$ be a principal ideal domain and let $F$ be its field of fractions. In the subsequent discussion, $\mathfrak{o}$ is usually taken to be the ring of integers $\mathbb{Z}$ or the ring of $p$-adic integers $\mathbb{Z}_{p}$, where $p$ is a prime number. A quadratic space over $F$ is a finite-dimensional $F$-vector space $V$ endowed with a symmetric bilinear form $B: V \times V \rightarrow F$. The function $Q: V \rightarrow F$ defined by $Q(v)=B(v, v)$ is a quadratic form on $V$. An o-lattice on $V$ is a finitely generated o-module $L$ which spans $V$. An element $a$ in $F$ is said to be represented by $L$ if there exists $v \in L$ such that $Q(v)=a$. To see the connection with number theory, let us fix a basis $\left\{v_{1}, \ldots, v_{n}\right\}$ of $L$. For $1 \leq i, j \leq n$, let $b_{i j}$ be $B\left(v_{i}, v_{j}\right)$. If $v=\sum_{i=1}^{n} x_{i} v_{i} \in L$, then

$$
Q(v)=\sum_{i, j=1}^{n} b_{i j} x_{i} x_{j}
$$

which is a quadratic form in the variables $x_{1}, \ldots, x_{n}$. Therefore, determining whether $a$ is represented by $L$ is the same as deciding if the quadratic diophantine equation

$$
\sum_{i, j=1}^{n} b_{i j} x_{i} x_{j}=a
$$

has a solution $\left(x_{1}, \ldots, x_{n}\right) \in \mathfrak{o}^{n}$. The set of elements of $F$ that are represented by $L$ is denoted by $Q(L)$.

Another o-lattice $M$ is said to be isometric to $L$, written $M \cong L$, if there is a bijective o-linear map $\sigma: M \rightarrow L$ which preserves the bilinear forms. Such a $\sigma$ is called an isometry from $M$ to $L$. Note that $Q(M)=Q(L)$ if $M \cong L$.

A $\mathbb{Z}$-lattice $L$ is called positive definite if $Q(v)>0$ for all nonzero $v$ in $L$. By Sylvester's law of inertia [12, $\S 61 \mathrm{~A}]$, the $\mathbb{R}$-space $\mathbb{R} \otimes V$ has an orthonormal basis with respect to $B$. In particular, $\mathbb{R} \otimes V$ is just the usual $n$-dimensional Euclidean space $\mathbb{R}^{n}$. Therefore, we may regard $V$ and $L$ as subsets of $\mathbb{R}^{n}$. In this case, for any $v \in V, Q(v)$ is the square-length of $v$, and $Q(L)$ is the same as the set $\mathcal{D}(L)$ defined in the previous section. For the remainder of this paper, the term lattice always refers to a positive definite $\mathbb{Z}$-lattice.

Now, suppose that $L$ is a lattice and that $V$ is the ambient quadratic space. Let $p$ be a prime number. The $p$-adic localization of $V$ is the space $V_{p}:=\mathbb{Q}_{p} \otimes V$ over the field of $p$-adic numbers $\mathbb{Q}_{p}$. The bilinear form on $V$ carries over to $V_{p}$ in the natural manner. Inside $V_{p}$ there is the $p$-adic localization $L_{p}$ of $L$, which is the $\mathbb{Z}_{p}$-lattice $\mathbb{Z}_{p} \otimes L$. The genus of $L$, denoted gen $(L)$, is the collection of all lattices $M \subseteq V$ such that $M_{p} \cong L_{p}$ for all primes $p$. Let $a$ be a positive integer. We say that $a$ is represented by the genus of $L$, written $a \in Q(\operatorname{gen}(L))$, if $a \in Q\left(L_{p}\right)$ for all primes $p$. This is equivalent to saying that there is a lattice $M \in \operatorname{gen}(L)$ which represents $a$; see [12, 102:5]. It is obvious that $Q(L) \subseteq Q(\operatorname{gen}(L))$, and $L$ is called regular when these two sets are equal. The authors of [11] exploit the regularity of some lattices in $\mathbb{R}^{3}$ to show that there is no measurable set which has the almost sure Steinhaus property on any one of those ternary regular lattices. It is known that not all lattices are regular; see [12, 102:6] for an example of a lattice which is not regular. However, when the rank of a lattice is at least 5 , it is almost regular, that is, $Q(\operatorname{gen}(L)) \backslash Q(L)$ is a finite set. 
Theorem 2.1 ([17]). Let $L$ be a positive definite lattice of rank at least 5 . There exists a constant $c(L)$ such that $a \in Q(L)$, provided $a \in Q(\operatorname{gen}(L))$ and $a>c(L)$.

The analytic proof by Tartakowsky that appeared in [17] does not produce or lead to any explicit estimate of the constant $c(L)$. An arithmetic proof given by HsiaKitaoka-Kneser in [6], which yields stronger and more generalized results, provides an effective procedure to produce numerical estimates of $c(L)$. Such a procedure is further detailed and carried out in [5].

Not every quaternary lattice is almost regular. For example, the lattice associated with the quadratic form $x_{1}^{2}+x_{2}^{2}+25 x_{3}^{2}+25 x_{4}^{2}$ represents all positive integers of the form $3 \cdot 2^{2 m}$ over $\mathbb{Z}_{p}$ for every prime $p$, but not over $\mathbb{Z} \mathbb{1}$ An integer $a$ is said to be primitively represented by $L$ if there exists $v \in L$ such that $Q(v)=a$ and $v$ can be extended to a basis of $L$. The set of all integers that are primitively represented by $L$ is denoted by $Q^{*}(L)$. The notations $Q^{*}\left(L_{p}\right)$ and $Q^{*}(\operatorname{gen}(L))$ are defined in the obvious manner.

Theorem 2.2. Let $L$ be a positive definite lattice of rank at least 4 . There exists a constant $c^{*}(L)$ such that $a \in Q^{*}(L)$, provided $a \in Q^{*}(\operatorname{gen}(L))$ and $a>c^{*}(L)$.

The above theorem appeared as Theorem 76 in Watson's book [18] without a proof; Watson commented that "This theorem can be deduced from results in the literature, obtained by analytic methods." Indeed, an equivalent version of Theorem 2.2 was proved analytically by Tartakowsky [17, and an alternative treatment was given by Pall and Ross [14. An arithmetic proof of Theorem 2.2 can be found in [2, Section 11.9]. An estimate on the size of $c^{*}(L)$ has been worked out in [15].

The situation when $L$ is ternary is more complicated. It is known that Theorem 2.2 does not hold for all ternary lattices, and a counterexample can be found in 2, page 228]. For an arbitrary lattice $L$, its genus is partitioned into finitely many subsets called spinor genera [12, §102]. The spinor genus that contains $L$ is denoted by $\operatorname{spn}(L)$. We say that an integer $a$ is primitively represented by $\operatorname{spn}(L)$, written $a \in Q^{*}(\operatorname{spn}(L))$, if there exists $M \in \operatorname{spn}(L)$ such that $a \in Q^{*}(M)$.

Theorem 2.3 (4]). Let $L$ be a positive definite lattice of rank at least 3 . There exists a constant $c^{\prime}(L)$ such that $a \in Q^{*}(L)$, provided $a \in Q^{*}(\operatorname{spn}(L))$ and $a>c^{\prime}(L)$.

It should be noted that at present the constant $c^{\prime}(L)$ for a ternary lattice $L$ cannot be made explicit without invoking the generalized Riemann hypothesis. Even if one assumes the Riemann hypothesis, the resulting estimate of $c^{\prime}(L)$ is usually too big for any practical use; see the remark in 13 .

\section{Proof of the main Result}

In this section, we shall give a proof of Proposition 1.4. We remind the readers that all lattices are positive definite $\mathbb{Z}$-lattices, as defined in the previous section. We begin by introducing some additional notations and terminologies. Let $L$ be a lattice. Suppose that $A$ is a matrix whose columns form a basis of $L$. The discriminant of $L$, denoted $d(L)$, is the determinant of $A A^{T}$. In particular, $d(L)=$ $v(L)^{2}$. The norm of $L$ is the fractional ideal $\mathfrak{n} L$ of $\mathbb{Z}$ generated by $Q(L)$. We say

\footnotetext{
${ }^{1}$ There is a typo in the example $x_{1}^{2}+x_{2}^{2}+5 x_{3}^{2}+5 x_{4}^{2}$ given in [2, page 235]; one should replace each ' 5 ' by ' 25 '. The argument presented there will still prevail verbatim.
} 
that $L$ is a-maximal [12, $\S 82 \mathrm{H}]$ if $\mathfrak{n} L \subseteq \mathfrak{a}$ and if for every lattice $K$ on $V$ which contains $L$ we have

$$
\mathfrak{n}(K) \subseteq \mathfrak{a} \Rightarrow K=L .
$$

Lemma 3.1. Every lattice has a sublattice whose genus has only one spinor genus.

Proof. Let $L$ be a lattice and let $\mathfrak{a}$ be its norm. By [12, 82:18], there is an $\mathfrak{a}$ maximal lattice $K$ on $V$ which contains $L$. Let $t$ be the group index $[K: L]$, which is a positive integer. Then $N:=t K$ is a $t^{2} \mathfrak{a}$-maximal sublattice of $L[12, \S 82 \mathrm{~J}]$. By [12. 102:10], there is only one spinor genus in the genus of $N$.

Lemma 3.2. Let $L$ be a lattice and let $N$ be a sublattice of $L$. If $M$ is a lattice such that $N \succ_{s} M$, then $L \succ_{s} M$.

Proof. It is clear because $v(N)=v(L)[L: N]$ and $Q(M) \subseteq Q(N) \subseteq Q(L)$.

Proof of Proposition 1.4. Let $L$ be an integral lattice of rank $n \geq 3$, and let $V$ be the ambient quadratic space which is embedded into $\mathbb{R}^{n}$. By virtue of Lemmas 3.1 and 3.2. we may assume that gen $(L)$ has only one spinor genus. Let $\left\{v_{1}, \ldots, v_{n}\right\}$ be a set of pairwise orthogonal vectors in $L$. By Dirichlet's theorem on primes in an arithmetic progression [16, page 73], there exists a prime $q$, not dividing $2 d(L) Q\left(v_{1}\right) \cdots Q\left(v_{n}\right)$, such that $q \equiv 1 \bmod p^{3}$ for all primes $p$ dividing $2 d(L)$. By the Local Square Theorem [12, 63:1], $q$ is the square of a unit in $\mathbb{Z}_{p}$ for every $p \mid 2 d(L)$.

Let $W$ be the $\mathbb{Q}$-space spanned by $v_{1}, \ldots, v_{n-1}, \sqrt{q} v_{n}$. For each $p \mid 2 d(L), \sqrt{q} \in$ $\mathbb{Q}_{p}$ and hence $W_{p}=V_{p}$. As a result, $W_{p}$ contains $L_{p}$ for all $p \mid 2 d(L)$. Let $J$ be the lattice spanned by $v_{1}, \ldots, v_{n-1}, \sqrt{q} v_{n}$, which is a lattice on $W$. By [12, 81:14], there is a lattice $N$ on $W$ such that

$$
N_{p}= \begin{cases}L_{p} & \text { if } p \mid 2 d(L), \\ J_{p} & \text { if } p \nmid 2 d(L) .\end{cases}
$$

Since $d(J)=Q\left(v_{1}\right) \cdots Q\left(v_{n-1}\right) \cdot q Q\left(v_{n}\right)$ and $q \nmid Q\left(v_{1}\right) \cdots q\left(v_{n}\right), \operatorname{ord}_{q}(d(N))=$ $\operatorname{ord}_{q}(d(J))=1$.

By Dirichlet's theorem again, there exists a prime $\ell>q$ which is the square of a unit in $\mathbb{Z}_{p}$ for every $p$ dividing $2 d(L)$. Let $M$ be the lattice $\sqrt{\ell} N$. We choose $\ell$ large enough that the nonzero minimum of the set $Q(M)$ is greater than the constant $c^{\prime}(L)$ obtained in Theorem 2.3. We claim that $L \succ_{s} M$.

Since $\operatorname{ord}_{q}(d(M))=\operatorname{ord}_{q}(d(N))=1$ and $q \nmid d(L), v(M) / v(L) \notin \mathbb{Q}$. Suppose that $a \in Q(M)$. Let $k$ be an integer such that $b=k^{-2} a$ is in $Q^{*}(M)$. For any $p \mid 2 d(L), \sqrt{\ell}$ is a unit in $\mathbb{Z}_{p}$; hence $L_{p}=M_{p}$ and $b \in Q^{*}\left(M_{p}\right)=Q^{*}\left(L_{p}\right)$. Let $p$ be a prime not dividing $2 d(L)$. Then $L_{p}$ is a unimodular $\mathbb{Z}_{p}$-lattice; see [12, $\S 82 \mathrm{G}$ ] for the definition and properties of unimodular $\mathbb{Z}_{p}$-lattices. If $b$ is a unit in $\mathbb{Z}_{p}$, then $b \in Q\left(L_{p}\right)$ [12, 92:1b]. Furthermore, any vector $v \in L_{p}$ with $Q(v)=b$ must be a part of an orthogonal basis of $L_{p}$ [12, 82:15a]. Therefore, $b \in Q^{*}\left(L_{p}\right)$. Now, suppose that $b$ is divisible by $p$. From the proof of [12, 92:1b], $L_{p}$ has an orthogonal basis $\left\{y_{1}, y_{2}, \ldots, y_{n}\right\}$ such that $Q\left(y_{1}\right)=-1$ and $Q\left(y_{2}\right)=1+b$. Then $Q\left(y_{1}+y_{2}\right)=b$, and clearly $y_{1}+y_{2}$ can be extended to a basis of $L_{p}$. Therefore $b \in Q^{*}\left(L_{p}\right)$ for all $p \nmid 2 d(L)$.

From the above discussion we see that $b \in Q^{*}(\operatorname{gen}(L))=Q^{*}(\operatorname{spn}(L))$. However, $b>c^{\prime}(L)$ because $b \in Q(M)$. By Theorem 2.3, $b \in Q^{*}(L)$ and hence $a \in Q(L)$. As a result, $L \succ_{s} M$ as claimed. 


\section{REFERENCES}

[1] J. Beck, On a lattice point problem of H. Steinhaus, Studia Sci. Math. Hung. 24 (1989), 263-268. MR1051154 (91i:11072)

[2] J. W. S. Cassels, Rational quadratic forms, Academic Press, 1978. MR0522835 (80m:10019)

[3] H. T. Croft, Three lattice-point problems of Steinhaus, Quart. J. Math. 33 (1982), 71-82. MR0689852 (85g:11051)

[4] W. Duke and R. Schulze-Pillot, Representation of integers by positive ternary quadratic forms and equidistribution of lattice points on ellipsoids, Invent. Math. 99 (1990), 49-57. MR.1029390 (90m:11051)

[5] J. S. Hsia and M. I. Icaza, Effective version of Tartakowky's theorem, Acta Arith. 89 (1999), 235-253. MR.1691853 (2000h:11031)

[6] J.S. Hsia, Y. Kitaoka, and M. Kneser, Representation by positive quadratic forms, J. Reine Angew. Math. 301 (1977), 132-141. MR0560499 (58:27758)

[7] S. Jackson and R. D. Mauldin, On a lattic problem of Steinhaus, J. Amer. Math. Soc. 15 (2002), 817-856. MR1915820 (2004b:52026)

[8] S. Jackson and R. D. Mauldin, Survey of the Steinhaus tiling problem, Bull. Symbolic Logic 9 (2003) no. 3, 335-361. MR2005953 (2004k:28011)

[9] M. N. Kolountzakis and M. Papadimitrakis, The Steinhaus tiling problem and the range of certain quadratic forms, Illinois J. Math. 46 (2002), 947-951. MR1951250 (2003m:52026)

[10] M. N. Kolountzakis and T. Wolff, On the Steinhaus tiling problem, Mathematika 46 (1999), 253-280. MR.1832620 (2002c:52024)

[11] R. D. Mauldin and A. Yingst, Comments about the Steinhaus tiling problem, Proc. Amer. Math. Soc. 131 (2003), 2071-2079. MR1963752 (2003k:28001)

[12] O.T. O'Meara, Introduction to quadratic forms, Springer Verlag, New York, 1963.

[13] K. Ono and K. Soundararajan, Ramanujan's ternary quadratic forms, Invent. Math. 130 (1997), 415-454. MR1483991 (99b:11036)

[14] A. Ross and G. Pall, An extension of a problem of Kloosterman, Amer. J. Math. 68 (1946), 59-65. MR0014378 (7:275b)

[15] R. Schulze-Pillot, On explicit versions of Tartakowsky's theorem, Arch. Math. (Basel) 77 (2001), no. 2, 129-137. MR 1842088 (2002i:11036)

[16] J. P. Serre, A Course in arithmetic, Springer-Verlag, 1973. MR0344216 (49:8956)

[17] W. Tartakowsky, Die Gesamtheit der Zahlen, die durch eine quadratische form $F\left(x_{1}, x_{2}, \ldots, x_{s}\right),(s \geq 4)$ darstellbar sind., Izv. Akad. Nauk SSSR (1929), 111-122, 165-196.

[18] G. L. Watson, Intgeral quadratic forms, Cambridge University Press, 1960. MR0118704 $(22: 9475)$

Department of Mathematics and Computer Science, Wesleyan University, MiddleTOWN, Connecticut 06459

E-mail address: wkchan@wesleyan.edu

Department of Mathematics, Box 311430, University of North Texas, Denton, Texas 76203

E-mail address: mauldin@unt.edu 